\title{
Vokabellernen (ohne) Vergessen (mit) Vokabellernstrategien
}

\section{Learning Vocabulary (without) Forgetting (with) Vocabulary Learning Strategies}

\begin{abstract}
The aim of this article is to describe the issue of effective vocabulary learning among the students of neophilological faculties as well as to provide an answer to the question on how to learn vocabulary effectively. To achieve this aim, two vocabulary learning strategies (the key-word method and learning by heart) were compared on the basis of empirical research. Moreover, the vocabulary learning style of the informants was described, the conclusions were drawn and recommendations given.
\end{abstract}

KEYWORDS: vocabulary learning, vocabulary learning strategies, key-word method, learning by heart.

\section{EINFÜHRUNG}

Der Erwerb einer Fremdsprache ist ein kontinuierlicher Prozess, in dem gleichzeitig mehrere sprachliche Kompetenzen wie Wortschatz, Grammatik sowie sprachliche Fertigkeiten (rezeptive wie: Lesen und Hören und produktive wie: Schreiben und Sprechen) herausgebildet und weiterentwickelt werden. Wilkins (1972: 111) nimmt an, dass Wortschatz der Grammatik übergeordnet ist, was er mit folgenden Worten hervorhebt: „Without grammar very little can be conveyed, without vocabulary nothing can be conveyed“. Daher sollte man in der Lehrerausbildung auf die mit der Wortschatzaneignung zusammenhängenden Kompetenzen fokussieren, zumal auf jedem der sprachlichen Niveaus Hunderte Vokabeln zu beherrschen sind und in zahlrei- 
chen Fällen die Wortschatzarbeit und die Entwicklung des Vokabellernens den Lernenden überlassen werden. Aus diesem Grund ist die Rolle der Steuerung seitens des Lehrers und der Selbststeuerung seitens der Lernenden bei der Wortschatzarbeit nicht zu vergessen. Das Ziel des vorliegenden Artikels ist es, auf die mit dem Gedächtnis verbundenen Prozesse, die Vokabellernstrategien und die Rolle der Lehrerausbildung in Bezug auf die Wortschatzaneignung näher einzugehen. Nach der theoretischen Darstellung des Forschungsstands werden Ergebnisse der empirischen Forschung präsentiert, wonach Schlussfolgerungen für Lehrer gezogen werden.

\section{ROLLE DES LEHRERS BEI DER WORTSCHATZARBEIT}

Bei der Wortschatzarbeit sollte der Lehrer sowohl bei der Selektion und Progression des Wortschatzes als auch bei dessen Kontrolle und Feedback helfen (Börner 2000: 32), weil er für solche Aktivitäten im FSU wie Bedeutungsvermittlung, Bedeutungserschließung, Übungs-, Wiederholungs-und Vertiefungsphasen des Unterrichts zuständig ist (vgl. Stork 2003: 14). Dies ist auch dem kognitiven Lehr-Lernmodell der Wortschatzarbeit von Börner (2000: 32) zu entnehmen. Darüber hinaus kommen auf den Lehrer im FSU noch zusätzliche, mit den Steuerungs- und Selbststeuerungsprozessen des Lehrens und Lernens zusammenhängende, autonom geprägte Funktionen zu: er sollte nicht nur Schwierigkeiten der Lernenden beim Wortschatzlernen erkennen, zu deren Überwindung anregen und sie dabei unterstützen (d.h. steuern). Vielmehr sollte er auch bei den Lernenden ihre individuellen Lernfähigkeiten herausbilden und sie von der Änderung eigener Lerngewohnheiten zugunsten der mit der Wortschatzarbeit verbundenen, besseren oder effektiveren Techniken und Strategien überzeugen (d.h. den Lernenden die Selbststeuerung des Lernprozesses ermöglichen) (Wilczyńska 1999: 119, 149). Da der Lehrprozess in der Regel zeitlich beschränkt ist (Fremdsprachenkurs, Fremdsprachenlernen in einer Schule, Studium etc.), erscheint die Herausbildung der Fähigkeit zum (zeitlich unbeschränkten) selbst gesteuerten Lernen umso wichtiger, zumal man dadurch auch dem Zustand, in dem der Lernprozess beendet wurde, d.h. der Fossilierung, entgegenwirken kann (Koeppel 2010: 9).

\section{GEDÄCHTNIS VERSUS VERGESSEN}

Bei jeder Form der Wortschatzaneignung (ungesteuert, gesteuert und selbst gesteuert) muss mit einem natürlichen Prozess gerechnet werden mit dem Vergessen. Dieser Prozess ist wiederum auf die Art und Funkti- 
onsweise des Gedächtnisses zurückzuführen. Engelkamp (1991: 6-8) fasst die verschiedenen Gedächtnismodelle zusammen und bildet drei große Gruppen: strukturalistische, funktionale und strukturell-funktionale Gedächtnistheorien. Eine funktionale Gedächtnistheorie ist z.B. die Theorie der Verarbeitungstiefe, nach der es nur einen Speicher gibt, wobei gilt, dass „je tiefer ein Stimulus verarbeitet wird, desto stärker und dauerhafter ist seine Gedächtnisspur, und desto besser kann erinnert werden" (Pethes, Ruchatz 2001: 616).

Ein Beispiel für eine strukturell-funktionale Gedächtnistheorie ist die Theorie der dualen Kodierung, die zwei Speicher voraussetzt: einen imaginalen, der die Verarbeitung nicht-sprachlicher Informationen ermöglicht, und einen sprachlichen (Paivio 1971: 179). Es wird angenommen, dass sich die Arbeit der beiden Speicher auf drei Ebenen vollzieht: auf der repräsentationalen, referentiellen und auf der assoziativen Ebene.

Die wichtigste und anerkannteste Gedächtnistheorie ist die strukturalistische Mehrspeichertheorie, nach der das Gedächtnis aus drei Speichern besteht, und zwar aus dem sensorischen Register, dem Kurzzeitgedächtnis und dem Langzeitgedächtnis. Diese drei Arten des Gedächtnisses spielen im Lernprozess eine bedeutende Rolle, wobei das Ziel des Fremdsprachenlernens (und des Lernens allgemein) die Überführung von Informationen ins Langzeitgedächtnis ist. Diese Informationen (z.B. Lexeme) müssen sehr oft mehrere Stadien durchgelaufen haben, bis ihre erfolgreiche Speicherung möglich wird (Meara 1996). ${ }^{1}$ Der Prozess des Vergessens kann sich in jedem Stadium vollziehen. Als Gründe werden v.a. Spurenzerfall, Interferenz, Nichtabrufbarkeit und Verdrängung angenommen. In Bezug auf die Wortschatzaneignung am wichtigsten scheint die Spurenzerfallstheorie, weil nur sie von einem Zerfall der Gedächtnisspuren ausgeht. Dies geschieht dann, wenn die Informationen nicht wiederholt werden, wodurch es schließlich zu deren Verlust kommt. Es wird angenommen, dass bis zu 80\% des gelernten Stoffes während der ersten 24 Stunden vergessen wird (Thornbury 2002: 26). Aus diesem Grund wird immer wieder empfohlen eine Wiederholung in immer größer werdenden Abständen einzusetzen. Rampillon (2003: 87) schlägt vor, dass die Wiederholung am Tag 2, 4, 16, 32 usw. stattfinden soll. Die Art der Wiederholung ist dabei für die Überbrückung des Vergessens auch nicht ohne Bedeutung - je vielfältiger das Lernen und die Wiederholung, desto größer der Behaltenserfolg. Soll das Lernen vielfältiger verlaufen und das Vergessen gemindert werden, so sollten Vokabellernstrategien zum Einsatz gebracht werden.

1 <http://www.lognostics.co.uk/vlibrary/meara1996c.pdf> (17.08.2011). 


\section{VOKABELLERNSTRATEGIEN}

Der Begriff „Lernstrategie“ wird von verschiedenen Autoren sehr unterschiedlich definiert. Für Friedrich/Mandl (1992: 6) bedeutet eine Strategie "eine Sequenz von Handlungen, mit der ein bestimmtes Ziel erreicht werden soll“, von Oxford (1990: 8) wird sie als „behaviours or actions which learners use to make language learning more successful, self-directed and enjoyable" bezeichnet. Andere Autoren verstehen unter Lernstrategien Bestandteile von Planungsprozessen oder auch Wissen über Strategien (Wenden, Rubin 1987: 6-7; Zimmermann 1997: 97). Da viele Autoren Lernstrategien aufgrund unterschiedlicher Kriterien analysiert haben, ist eine große Zahl von Klassifikationen entstanden - Neuner-Anfindsen (2005: 118) spricht sogar von 20 Klassifikationssystemen. Für den vorliegenden Artikel sind aber nur diese am wichtigsten, die direkt auf das Vokabellernen Bezug nehmen, d.h. Speicher- und Abrufstrategien.

In Bezug auf die Speicherstrategien unterscheidet Neveling (2004: 74) die Strategien, die zur Ordnung, Elaboration und Wiederholung des Stoffes dienen. Die Informationen können hierarchisch, merkmalsorientiert, sachorientiert, syntagmatisch, phonetisch, affektiv, interlingual (L1/L3) und nach Wortfamilien geordnet werden (ebd.). Solche Verfahren erleichtern die Arbeit des Gedächtnisses und ermöglichen die Herausbildung der dauerhafteren Verbindungen zwischen den jeweiligen Elementen. Die zweite Stufe ist die Elaboration, die sich dann anwenden lässt, wenn es sich um die Speicherung von wenig sinnbehafteten Einheiten handelt. Diese Einheiten werden dadurch mit sinnreichen Elementen in Verbindung gesetzt, was wiederum den Speicherprozess fördert (Neveling 2004: 75). Die Art der Verbindung ist von bestimmten Elaborationsstrategien abhängig (unterschieden werden: kinästhetische, emotionale, episodische, kontextuelle und sensorische Elaborationsstrategien). Somit hat man im Fall kinästhetischer Elaborationsstrategien mit einer motorisch-kognitiven Verbindung von Aktivitäten $\mathrm{zu}$ tun (Wörter können mit inhaltsbegleitenden Bewegungen, Gesten, Rollenspielen verbunden oder von den Lernenden aufgeschrieben werden) (Neveling 2004: 76). Im Fall emotionaler Elaborationsstrategien handelt es sich um das Verbinden von Wörtern mit eigenen Emotionen, mit dem Ort des Lernens etc. Sollten episodische Strategien im Einsatz sein, so kann man einige folgender auswählen: Nacherzählen, Paraphrasieren, Zusammenfassen, Notizenmachen. Schließlich bietet sich den Lernenden auch eine Gruppe von sensorischen Strategien, zu denen vor allem auditive (wie z.B. Vokabel singen, Reime, Merkverse, Schlüsselwort) und visuelle Strategien (Zeichnungen, Abkürzungen, Wortikone, Loci-Technik) gehören (ebd.). Nach der Elaboration des Materials kommt es zu dessen Einprägen und zur Wieder- 
holung. Dies lässt sich durch intensives Anschauen, lautes oder leises Lesen/Vorsprechen, wiederholtes Aufschreiben, Bilden neuer Kontexte, mündliche Abfragung erreichen, was wiederum systematisch, gezielt, selektiv und in Intervallen verlaufen sollte (Neveling 2004: 77). Alle diese, in drei Gruppen gefassten Speicherstrategien, die zur Ordnung, Elaboration, Einprägung und Wiederholung des Materials angewandt werden, haben zum Ziel, den Speicherprozess zu erleichtern, d.h. den Übergang von Informationen ins Langzeitgedächtnis zu fördern und somit auf den Lernprozess positiv einzuwirken.

Die Abrufstrategien hingegen finden ihre Anwendung, wenn das gelernte Material im Moment nicht zugänglich ist. Sie werden von Neveling (2004: 77) in zwei Untergruppen geteilt: in interaktive, wie z.B. fokussierendes Warten, metasprachliche Appelle, Abfragenlassen und in non-interaktive, in denen Ordnungsnetze (hierarchisch, merkmalsorientiert etc.) und Elaborationselemente (kinästhetisch, emotional etc.) aktiviert werden.

Strategien bieten den Lernenden eine Vielfalt von Möglichkeiten, den Lernprozess interessanter, effizienter zu gestalten (Aktivierung des Langzeitgedächtnisses). Aus der eigenen empirischen Untersuchung (vgl. den nächsten Abschnitt) geht aber hervor, dass nur wenige Vokabellernstrategien von den Lernenden benutzt werden. Ihr Wissen darüber ist nicht zufriedenstellend, woraus man schlussfolgern kann, dass sie mit den Strategien nicht ausreichend vertraut sind. Morfeld (1998: 69) stellt Folgendes fest: „die Studierenden besitzen wenig Vorwissen in Bezug auf Lernstrategien, Lerntechniken und haben Schwierigkeiten damit, zwischen Inhalts- und Strategievermittlung zu unterscheiden". Daraus ergibt sich die Notwendigkeit für die Lehrenden, sich Wissen über die Strategien und ihre Vermittlung anzueignen, sodass sie ihrerseits in der Lage sind, den Lernenden einige Strategien zur Verfügung zu stellen.

\section{EMPIRISCHE UNTERSUCHUNG}

Die empirische Untersuchung umfasst die Durchführung eines Experiments und einer quantitativen Befragung. In dem Experiment wurden zwei Vokabellernstrategien bezüglich ihrer Effizienz empirisch erprobt. Es waren die Schlüsselwortmethode, ${ }^{2}$ die nicht sehr bekannt ist aber als eine effiziente Strategie gilt,

${ }^{2}$ Das Lernen mithilfe der Schlüsselwortmethode läuft in zwei Schritten ab. Zuerst ruft man ein Wort in der Muttersprache (bzw. in einer anderen Sprache) hervor, dessen Klang entweder identisch mit oder ähnlich dem neuen, zu lernenden Wort ist (dieses Wort ist dann das Schlüsselwort). Ferner verbindet man die Bedeutung des unbekannten Wortes mit der Bedeutung des Schlüsselwortes, indem eine Assoziation oder ein mentales Bild entsteht. 
und das Auswendiglernen, das im Gegenteil zur Schlüsselwortmethode häufig verwendet, aber als weniger effektiv eingeschätzt wird. In der quantitativen Befragung ging es hingegen um die Erforschung der Vokabellerngewohnheiten, der Anwendung von Vokabellernstrategien und der Art des Wiederholens unter Studierenden der philologischen Fakultäten in Poznań.

Das Experiment wurde im Institut für Angewandte Linguistik in Poznań durchgeführt. Als Zielgruppe wurde eine Gruppe des ersten Studienjahres ausgewählt, die am Anfang 18 Personen zählte. Das Lebensalter der Versuchspersonen lag zwischen 18 und 21 Jahren. Alle lernten Deutsch seit mindestens 4 Jahren. Den TeilnehmerInnen wurde erläutert, worin das Experiment bestehen würde (das Ziel, die Dauer, die einzusetzenden Materialien etc.). Es fanden 4 Sitzungen statt. In der ersten Sitzung wurden kurze Fragebögen zur Gewinnung statistischer Daten verteilt; die Schlüsselwortmethode wurde erklärt, das erste Wortset verteilt (10 deutsche Substantive samt Artikeln ${ }^{3}$ und ihren polnischen Entsprechungen). Nach dem zehnminütigen Einprägen bekamen die Versuchspersonen den Kontrolltest I, der das Kurzzeitgedächtnis überprüfen sollte. Nach einer Woche folgte der Kontrolltest II, nach zwei Wochen der Kontrolltest III. ${ }^{4}$ Bei der Evaluation der Kontrolltests wurde nach dem Richtig-Falsch-Verfahren vorgegangen, d.h. war entweder der Artikel oder das Substantiv falsch angegeben, wurde die Antwort als falsch betrachtet; waren der Artikel und das Substantiv korrekt, galt die Antwort als richtig. Nach dem Kontrolltest I bekamen die TeilnehmerInnen einen zusätzlichen Fragebogen zur Überprüfung der Bekanntheit der präsentierten Vokabellernstrategie und deren früheren Erfahrung. Darüber hinaus wurden auch die Anzahl der mittels der Strategie gelernten Vokabeln und eventuelle Schwierigkeiten erfragt. In der zweiten Sitzung wurde der Kontrolltest II zur Schlüsselwortmethode verteilt, wonach die zweite Vokabellernstrategie (Auswendiglernen) erläutert und das zweite Wortset ${ }^{5}$ ausgeteilt wurde. Es folgten der Kontrolltest I und ein Fragebogen

${ }^{3}$ Die Substantive wurden einem medizinischen Fachsprachewörterbuch entnommen, um für die TeilnehmerInnen unbekannt zu sein. Bei deren Auswahl wurde nach folgenden Kriterien vorgegangen: gleichen Silbenzahl und Variiertheit der auftretenden Vokale und Konsonanten. Das Wortset wurde nach dem Einprägen der Vokabeln wieder eingesammelt. Ein Beispiel für ein Wortpaar: die Schwäre - ropień.

${ }^{4}$ Der Kontrolltest II sollte das "Mittelzeitgedächtnis" (d.h. das sich zwischen dem Kurzund Langzeitgedächtnis platzierte Gedächtnis), der Kontrolltest III das Langzeitgedächtnis prüfen. Jeder Kontrolltest umfasste 10 aufgelistete polnische Substantive, zu denen die TeilnehmerInnen die deutschen Entsprechungen schreiben sollten. Zwecks Vermeidung des Positionseffekts waren die Substantive jeweils in einer anderen Reihenfolge angegeben.

${ }^{5}$ Das zweite Wortset umfasste auch 10 Substantive (diesmal einem technischen Fachsprachewörterbuch entnommen) samt Artikeln und polnischen Entsprechungen. Ein Beispiel für ein Wortpaar: das Schälen - podorywka. 
mit zusätzlichen Fragen zum Auswendiglernen. In der dritten Sitzung fanden nur Kontrolltests statt. In der vierten Sitzung bekamen die TeilnehmerInnen außer den letzten Kontrolltest zur Strategie „Auswendiglernen“ noch einen Bogen mit abschließenden Fragen zur Beurteilung der Effektivität der beiden Vokabellernstrategien und zur potenziellen Anwendung in der Zukunft. Wegen der Abwesenheit mancher Versuchspersonen während aller Sitzungen, ist leider die Zahl der TeilnehmerInnen von 18 auf 13 gesunken (d.h. $\mathrm{n}=13) .{ }^{6}$ Aus diesem Grund werden die Ergebnisse des Experiments mit den Ergebnissen des Experiments von Stork (2003) verglichen, in dem insgesamt vier Vokabellernstrategien empirisch erprobt wurden, wobei die Anzahl der Versuchspersonen 41 betrug $(n=41)$. Zwecks Veranschaulichung wurden die Daten prozentuell ausgerechnet und mithilfe von Kurvendiagrammen dargestellt. ${ }^{7}$

Aus den obigen Diagrammen lässt sich entnehmen, wie viel Prozent des Stoffes mit welcher Vokabellernstrategie nach jedem Kontrolltest memoriert und korrekt angegeben wurde. Die beiden Diagramme zeigen die Überlegenheit der Schüsselwortmethode gegenüber dem Auswendiglernen. Aus dem ersten Diagramm geht hervor, dass gleich nach dem Einprägen (nach dem Kontrolltest I) bessere Resultate mithilfe von Auswendiglernen (95\% des Stoffes) erzielt wurden, während mit der Schlüsselwortmethode nur 90,1\% erreicht wurden. Dies bedeutet, dass Auswendiglernen besser für das effiziente und schnelle Einprägen geeignet ist. Eine Rolle spielt jedoch die Menge des zu lernenden Stoffes. Im Fall von $10 \mathrm{zu}$ lernenden Einheiten lag die Effizienz des Lernens bei 95\% (Abb.1), als es aber 20 Einheiten zum Lernen gab, sank die Effizienz des Auswendiglernens auf 77,2\% (Abb.2) und fiel schlechter als bei der Schlüsselwortmethode aus. Im Laufe der Zeit sank die Effizienz der beiden Vokabellernstrategien. ${ }^{8}$ Bezüglich des „Mittelzeitgedächtnisses" schnitt die Schlüsselwortmethode in der eigenen Untersuchung zweimal so gut wie Auswendiglernen ab (jeweils $68 \%$ und $34 \%$ ), in der Untersuchung von Stork fast zweimal so gut (38,2\% und 20,1\%) (Stork 2003: 150-152). Nach dem Kontrolltest III sahen die Ergebnisse der eigenen Untersuchung ähnlich aus (mit der Schlüsselwortmethode konnten die Ver-

${ }^{6}$ Die mindestens einmal abwesenden Personen konnten im Experiment nicht berücksichtigt werden, weil dann die Ergebnisse unvollständig und inkohärent gewesen wären.

7 Es bestehen allerdings einige Unterschiede zwischen den beiden Untersuchungen, z.B. in der Zahl: der geprüften Vokabellernstrategien (2 und 4), der TeilnehmerInnen (13 und 41), der zu lernenden Vokabeln (10 und 20) sowie in der Art der Vokabeln (authentische und künstlich konzipierte). Die Vergessenskurven zur Untersuchung von Stork wurden mithilfe von den in ihrer Dissertation dargestellten Mittelwerten erstellt.

${ }^{8}$ In der Zwischenzeit waren aufgrund des Einsammelns der Wortsets keine Wiederholungen der Vokabeln möglich. 


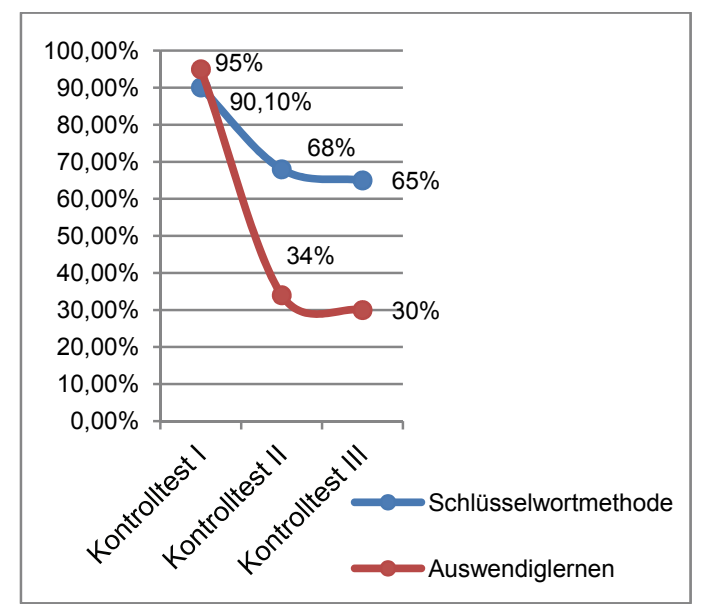

Abb. 1. Vergessenskurven zur eigenen Untersuchung

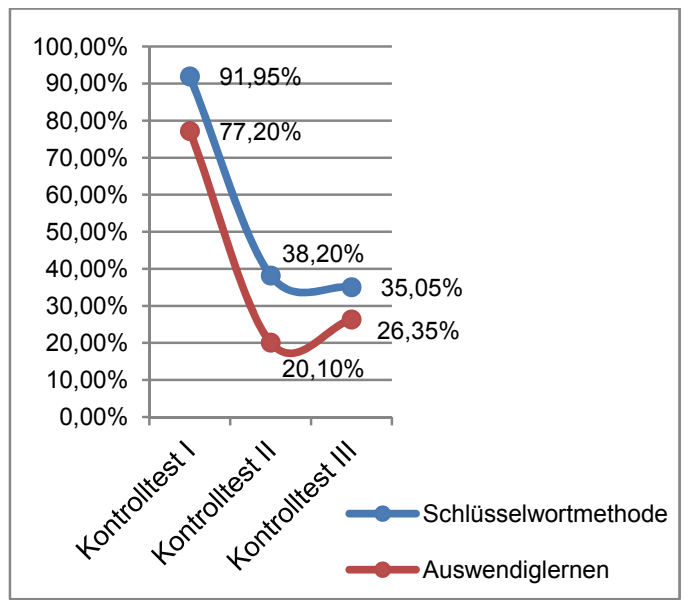

Abb. 2. Vergessenskurven zur Untersuchung von Stork (2003)

suchspersonen um 35\% mehr Stoff einprägen, d.h. über zweimal so viel wie mit dem Auswendiglernen). In Storks Untersuchung war die Diskrepanz zwischen beiden Strategien viel niedriger und betrug nur 8,7\% (Stork 2003: 152-154). ${ }^{9}$

${ }^{9}$ Dies ist aber auf die Zahl der geprüften Vokabellernstrategien und auf die Zahl der zu lernenden Vokabeln zurückzuführen. Die Schlüsselwortmethode wurde von Stork in Gruppe A als dritte und in Gruppe B als zweite getestet und das bedeutet, dass die Versuchspersonen vorher 40 oder 20 Vokabeln zu memorieren hatten (das konnte für die niedrigere Effizienz dieser Strategie im Vergleich zur eigenen Forschung ein Grund sein). Das Auswendiglernen schnitt aber in Storks Untersuchung noch schlechter ab, obwohl es am Anfang, d.h. als erste Strategie getestet wurde, was auf die Ineffektivität dieser Strategie hindeuten lässt. 
Aufgrund beider Untersuchungen kann geschlussfolgert werden, dass sich mit Auswendiglernen gute Ergebnisse erzielen lassen, wenn es sich um das Memorieren von nicht vielen Informationen handelt. Sie sind aber gleich nach dem Lernen zu erwarten (die Informationen werden nur im Kurzzeitgedächtnis gespeichert). Die Schlüsselwortmethode ermöglicht dahingegen die Speicherung im Langzeitgedächtnis, die die Abrufbarkeit von gelernten Informationen ermöglicht und erleichtert.

Die Zielgruppe der quantitativen Untersuchung bildeten Studierende, die Angewandte Linguistik (30), Germanistik (31) oder Deutsch als Fremdsprache im Fremdsprachenkolleg (29 Personen) studierten. Insgesamt belief sich die Zahl auf 90 Probanden. Der Fragebogen umfasste 30 in vier Fragenkomplexe eingeteilte Fragen und zwar: statistische Daten, Vokabellerngewohnheiten, Vokabellernstrategien, Wiederholen. Die Ergebnisse werden an dieser Stelle verallgemeinernd in Bezug auf die drei letzteren Fragenkomplexe präsentiert, ohne dass man sich jedoch mit jeder Frage detailliert auseinandersetzt (für die detaillierte Analyse der Untersuchungsergebnisse mit allen Balkendiagrammen vgl. Cudzich 2010).

In Bezug auf Vokabellerngewohnheiten gaben 78\% der Befragten an, Vokabeln gern zu lernen. $\mathrm{Zu}$ den wichtigsten Motivationsfaktoren gehören: Möglichkeit der Wortschatzerweiterung, pragmatische Gründe (wie z.B. Erweiterung der sprachlichen Kenntnisse, schönere Ausdrucksweise, bessere und einfachere Kommunikation) und schließlich Spaß beim Lernen. Der restliche Teil der Befragten lernt Vokabeln ungern, weil dies ein langweiliges und uneffektives Verfahren ist, dem man viel Zeit widmet und dennoch viele Vokabeln schnell vergessen werden. Des Weiteren lernt ein Drittel der Befragten zwischen 0 und 15 Vokabeln pro Woche. 27\% lernen zwischen 16 und 30 Vokabeln. Über 50 Vokabeln werden nur von 9\% der Befragten gelernt. Es muss noch unterstrichen werden, dass zwei Drittel der Probanden Vokabeln unregelmäßig und nur 13\% regelmäßig lernen. Bei 19\% findet das regelmäßige Vokabellernen nur vor Tests statt.

Die Bekanntheit und Häufigkeit der Anwendung vieler Vokabellernstrategien wurden mithilfe des dritten Fragenkomplexes erforscht. Aufgrund der gewonnenen Daten ließen sich die Vokabellernstrategien in drei Gruppen einteilen, und zwar: in die gut bekannten und häufig gebrauchten Strategien, in die bekannten und von einigen Probanden gebrauchten und in die weniger bekannten und/oder von den meisten Probanden selten oder auch gar nicht gebrauchten Strategien. Somit gehören zu der ersten Gruppe folgende Strategien: Auswendiglernen (Zuhaltemethode), Schreiben von zweispaltigen, deutsch-polnischen Listen, lautes Vorlesen der Texte/Artikel/ /Vokabeln, nocheinmaliges Lesen der Texte, Beschreibung der Welt auf Deutsch, Aufschreiben von schwierigsten Vokabeln, Ordnen von Vokabeln 
nach Synonymen und Sachfeldern, Generierung von vertrauten Bildern beim Lernen von konkreten Vokabeln und Benutzung von deutschen Medien (Zeitungen, Zeitschriften, Bücher, Fernsehen, Lieder, Filme). Manchmal oder selten werden dahingegen folgende Strategien eingesetzt: (lautes) Sprechen im Kontext bei festen Ausdrücken, Aufschreiben von Beispielsätzen, Ordnen von Vokabeln nach Wortfamilien und Antonymen. Die dritte Gruppe umfasst die selten oder gar nicht gebrauchten Strategien. Es sind: Aufnehmen der Texte auf Kassette oder CD und Überprüfung der Aussprache und Betonung, Führen eines Vokabelheftes oder einer Vokabellernkartei, Erfindung von (komischen) Geschichten beim Lernen der nicht zusammenhängenden Wörter und Ausführen von Bewegungen beim Lernen von Vokabeln, die diese Bewegungen bezeichnen.

Was die Wiederholungsgewohnheiten der Befragten betrifft, erwies sich, dass Vokabeln vor allem mündlich (von 88\%) und nur manchmal (von 61\% Befragten) wiederholt werden. Darüber hinaus wird das von Rampillon vorgeschlagene Wiederholungssystem selten (betrifft 43\% der Befragten) oder gar nicht (28\%) verwendet. Das zwecks Wiederholung nochmalige Aufschreiben von Wörtern in veränderter Reihenfolge findet von der Mehrheit der Probanden selten (über ein Drittel) oder gar nicht (23\%) Anwendung. Fast ein Drittel gab an, diese Strategie manchmal anzuwenden - nur bei $15 \%$ passiert das sehr oft. Noch seltener findet das Abfragen statt (selten von $40 \%$, manchmal von $28 \%$, nie von $20 \%$ und sehr oft von nur $12 \%$ der Befragten). Die überwältigende Mehrheit lässt sich von anderen Personen mündlich abfragen (betrifft $72 \%$ ).

Die quantitative Befragung zeigt, dass die Studierenden über manche Vokabellernstrategien verfügen (die erste und teilweise die zweite Gruppe). $\mathrm{Ob}$ das aber für Studenten neuphilologischer Studiengänge, für zukünftige Lehrer ausreichend und zufriedenstellend ist, bleibt trotz der eigenen Erfolgseinschätzung beim Vokabellernen unter den Probanden offen, in der $46 \%$ der Befragten ihren Erfolg als hinreichend, $41 \%$ als groß und $6 \%$ als verbesserungswürdig einschätzten. Dies deutet auf die Notwendigkeit hin, sich mit Vokabellernstrategien im FSU zu befassen, denn viele von ihnen werden selten oder überhaupt nicht angewandt, was auch auf ihre Unbekanntheit zurückzuführen ist. ${ }^{10}$ Die Ergebnisse der Experimente beweisen hingegen die Effektivität der nicht überall bekannten Strategie, der Schlüsselwortmethode, gegenüber dem am häufigsten angewandten Auswendiglernen.

10 39\% der Versuchspersonen (betrifft das eigene Experiment) und 65\% (betrifft Storks Experiment, Stork 2003: 154) waren mit der Schlüsselwortmethode früher, d.h. vor dem Experiment, nicht vertraut. 


\section{SCHLUSSFOLGERUNGEN FÜR LEHRER}

Um zum selbst gesteuerten Fremdsprachenerwerb multikompetent ausgebildet zu werden, brauchen die Lernenden einer Fremdsprache ein breites Repertoire von Handwerkszeug, das von ihnen zeitlich unbegrenzt, zielgerichtet und aufgabenorientiert angewandt werden kann. Es handelt sich um Vokabellernstrategien, deren Vermittlung die Aufgabe des Lehrers sein sollte. Die Vokabellernstrategien können in den FSU einbezogen werden, sodass die Lernenden die Chance bekommen, sie kennenzulernen, zu erproben, über ihre eigenen Lernmethoden, deren Effizienz zu reflektieren und vielleicht einige von den präsentierten Strategien zur Anwendung zu bringen. Dies setzt aber eine gute Lehrerausbildung in Bezug auf Lernstrategien voraus. Einerseits sollte der Lehrer über das kognitive Wissen über Lernstrategien verfügen, andererseits ist aber auch das prozedurale Wissen erforderlich, d.h. die Strategien müssen zuerst vom Lehrer selbst erprobt werden, um dann den Lernenden beigebracht werden zu können. Da jedoch wenige Vokabellernstrategien bezüglich ihrer Effizienz zurzeit erforscht wurden, wäre es sinnvoll, noch viele andere empirisch zu untersuchen. Somit könnte eine Liste von meist effektivsten Vokabellernstrategien entstehen, die wiederum von dem Lehrer durch ein dazu speziell erarbeitetes, auf die Bedürfnisse der Lernenden, ihr Alter, Fremdspracheniveau, ihre Motivation und vielleicht auch ihr Geschlecht zugeschnittenes Vokabellerntraining vermittelbar wäre. Natürlich müssten dann die Lehrer imstande sein, solch ein Training vorzubereiten und durchzuführen, d.h. manche Strategien regelmäßig in den Unterricht einzubeziehen und sie gemeinsam mit den Lernenden zu erproben. Und wie es sich im Experiment herausstellte, lohnen sich solche Versuche, weil die Schlüsselwortmethode von $69 \%$ der Versuchspersonen als sehr effektiv oder effektiv eingeschätzt wurde (jeweils 54 und 15\%), zumal 69\% der Versuchspersonen vorhaben, diese Strategie sehr oft oder manchmal anzuwenden (jeweils 38 und 31\%).

Empfehlenswert wäre auch, den Lernenden beim Wiederholen des Stoffes zu helfen, sodass die Effizienz ihres Lernens auf einem hohen Niveau gehalten, ihre Motivation dadurch gesteigert wird und die Lernenden schließlich auf das autonome Lernen vorbereitet werden, was eine der wichtigsten Aufgaben eines Lehrers ist. Dazu eigneten sich folgende Formen: häufigere Evaluation des Lernprozesses, das gemeinsame, häufigere Wiederholen des Materials in Form von Spielen, zusätzlichen Übungen und Aufgaben o.ä. Es muss aber betont werden, dass diese Aktivitäten allmählich den Lernenden übergeben werden sollten. 


\section{LITERATURVERZEICHNIS}

Börner, W., 2000. Didaktik und Methodik der Wortschatzarbeit: Bestandsaufnahme und Perspektiven. In: Kühn, P. (Hrsg.). Wortschatzarbeit in der Diskussion. Germanistische Linguistik Bd. 5, 29-56.

Cudzich, B., 2010. Effiziente Wortschatzarbeit im fortgeschrittenen Fremdsprachenunterricht? Das Fallbeispiel DaF-Studium in Polen. Unveröffentlichte Magisterarbeit. Adam Mickiewicz Universität. Poznań.

Engelkamp, J., 1991. Das menschliche Gedächtnis. Das Erinnern von Sprache, Bildern und Handlungen. Göttingen: Hogrefe.

Friedrich, H.F., Mandl, H., 1992. Lern- und Denkstrategien. Analyse und Intervention. Göttingen: Hogrefe.

Koeppel, R., 2010. Deutsch als Fremdsprache - Spracherwerblich reflektierte Unterrichtspraxis. Baltmannsweiler: Schneider Verlag Hohengehren.

Meara, P., 1996. The vocabulary knowledge framework. <http://www.lognostics.co.uk/vlibrary/ meara1996c.pdf> (17.08.2011).

Morfeld, P., 1998. Wissend lernen = effektiver lernen? Vokabellerntraining im Anfängerunterricht Englisch an der Volkshochschule. Tübingen: Gunter Narr Verlag.

Neuner-Anfindsen, S., 2005. Fremdsprachenlernen und Lernerautonomie. Sprachlernbewusstsein, Lernprozessorganisation und Lernstrategien zum Wortschatzlernen in Deutsch als Fremdsprache. Bd. 1., Baltmannsweiler: Schneider Verlag Hohengehren.

Neveling, C., 2004. Wörterlernen mit Wörternetzen. Eine Untersuchung zu Wörternetzen als Lernstrategie und als Forschungsverfahren. Tübingen: Gunter Narr Verlag.

Oxford, R.L., 1990. Language Learning Strategies. What Every Teacher Should Know. Boston: Heinle \& Heinle.

Paivio, A., 1971. Imagery and Verbal Processes. New York: Holt, Rinehart \& Winston.

Pethes, N., Ruchatz, J., 2001. Gedächtnis und Erinnerung. Ein interdisziplinäres Lexikon. Hamburg: Rowohlt Taschenbuch Verlag.

Rampillon, U., 2003. Better in Englisch. Unterrichtshilfen für Lehrer und Eltern. Hannover: Impressum.

Stork, A., 2003. Vokabellernen. Eine Untersuchung zur Effizienz von Vokabellernstrategien. Tübingen: Gunther Narr Verlag.

Thornbury, S., 2002. How to teach vocabulary. Harlow: Longman.

Wenden, A., Joan, R. (Hrsg.), 1987. Learner Strategies in Language Learning. Englewood Cliffs: Prentice Hall.

Wilczyńska, W., 1999. Uczyć się czy być nauczanym? O autonomii w przyswajaniu języka obcego. Warszawa-Poznań: Wydawnictwo Naukowe PWN.

Wilkins, D., 1972. Linguistics in Language Teaching. London: Edward Arnold.

Zimmermann, G., 1997. Anmerkungen zum Strategienkonzept. In: Rampillon, U., Zimmermann, G. (Hrsg.). Strategien und Techniken beim Erwerb fremder Sprachen. Ismaning: Hueber, 95-113. 